Swarthmore College

Works

8-1-2009

\title{
Modelling, Design And Diagnostics For A Photoionised Plasma Experiment
}

\author{
I. M. Hall \\ T. Turmaz \\ R. C. Mancini \\ J. E. Bailey \\ G. Rochau
}

See next page for additional authors

Follow this and additional works at: https://works.swarthmore.edu/fac-physics

Part of the Astrophysics and Astronomy Commons

Let us know how access to these works benefits you

\section{Recommended Citation}

I. M. Hall; T. Turmaz; R. C. Mancini; J. E. Bailey; G. Rochau; Michael Jonathan Rosenberg , '08; David H. Cohen; I. Golovkin; J. J. MacFarlane; M. E. Sherril; J. Abdallah; R. F. Heeter; M. E. Foord; S. H. Glenzer; and H. A. Scott. (2009). "Modelling, Design And Diagnostics For A Photoionised Plasma Experiment". Astrophysics And Space Science. Volume 322, Issue 1-4. 117-121. DOI: 10.1007/s10509-008-9930-4 https://works.swarthmore.edu/fac-physics/60

This work is brought to you for free by Swarthmore College Libraries' Works. It has been accepted for inclusion in Physics \& Astronomy Faculty Works by an authorized administrator of Works. For more information, please contact myworks@swarthmore.edu. 


\section{Authors}

I. M. Hall; T. Turmaz; R. C. Mancini; J. E. Bailey; G. Rochau; Michael Jonathan Rosenberg , '08; David H.

Cohen; I. Golovkin; J. J. MacFarlane; M. E. Sherril; J. Abdallah; R. F. Heeter; M. E. Foord; S. H. Glenzer; and H. A. Scott 


\title{
Modelling, Design and Diagnostics For A Photoionised Plasma Experiment
}

\author{
I. M. Hall ${ }^{1}$ and T. Turmaz ${ }^{1}$ and R. C. Mancini ${ }^{1}$, \\ J. E. Bailey ${ }^{2}$ and G. Rochau ${ }^{2}$, M. Rosenberg ${ }^{3}$ and \\ D. Cohen ${ }^{3}$, I. Golovkin ${ }^{4}$ and J. MacFarlane ${ }^{4}, M$. \\ Sherril $^{5}$ and J. Abdallah ${ }^{5}$, R. Heeter ${ }^{6}$ and M. \\ Foord $^{6}$ and S. Glenzer ${ }^{6}$ and H. A. Scott ${ }^{6}$
}

(C) Springer-Verlag $\bullet \bullet \bullet \bullet$

\begin{abstract}
Photoionised plasmas are common in astrophysical environments and new high resolution spectra from such sources have been recorded in recent years by the Chandra and XMM-Newton satellites. These provide a wealth of spectroscopic information and have motivated recent efforts aimed at obtaining a detailed understanding of the atomic-kinetic and radiative characteristics of photoionised plasmas. The Z-pinch facility at the Sandia National Laboratories is the most powerful terrestrial source of X-rays and provides an opportunity to produce photoionised plasmas in a well characterised radiation environment. We present modelling work and experimental design considerations for a forthcoming experiment at Sandia in which X-rays from a collapsing Z-pinch will be used to photoionise low density neon contained in a gascell. View factor calculations were used to evaluate the radiation environment at the gascell; the hydrodynamic characteristics of the gascell were examined using the Helios-CR code, in particular looking at the heating, temperature and ionisation of the neon and the absorption of radiation. Emission and absorption spectra were also computed, giving estimates of spectra likely to be observed experimentally.
\end{abstract}

I. M. Hall and T. Turmaz and R. C. Mancini, J. E. Bailey and G. Rochau, M. Rosenberg and D. Cohen, I. Golovkin and J. MacFarlane, M. Sherril and J. Abdallah, R. Heeter and M. Foord and S. Glenzer and H. A. Scott

${ }^{1}$ University of Nevada, Reno

${ }^{2}$ Sandia National Laboratories

${ }^{3}$ Swarthmore College

${ }^{4}$ Prism Computational Sciences

${ }^{5}$ Los Alamos National Laboratory

${ }^{6}$ Lawrence Livermore National Laboratory

\section{Introduction}

Photoionised plasmas are common in astrophysical environments, typically occurring near strong sources of $\mathrm{X}$-rays such as active galactic nuclei and X-ray binaries. In recent years, the Chandra and XMM-Newton satellites have recorded high resolution spectra from astrophysical photoionised plasmas. These contain a wealth of spectroscopic information and have motivated recent efforts aimed at obtaining a detailed understanding of the atomic-kinetic and radiative characteristics of photoionised plasmas. However, models used to interpret spectra from astrophysical plasmas are complex and require many parameters to be known including the geometry of the system, gas composition and density distribution, and the energy distribution of the ionising $\mathrm{X}$-ray source. Uncertainties inherent in these parameters will affect the validity of the results obtained from the spectral analysis.

In photoionised plasmas, the radiation field plays a dominant role in the atomic-kinetic behaviour, and collisional processes do not strongly influence the ionisation balance. In contrast, the population distribution in most laboratory produced plasmas is largely determined by collisional processes. Conditions of strong ionising radiation field and low collisionality are difficult to achieve experimentally, and there have been relatively few studies of terrestrial photoionised plasmas $(1 ; 2 ; 3)$. The Z-pinch facility at Sandia National Laboratories is currently the most powerful terrestrial source of X-rays, and is capable of producing a photionised plasma.

In this paper, we present modelling work and experimental design considerations for a forthcoming experiment at Sandia in which X-rays from a collapsing Z-pinch plasma will be used to photoionise low density neon contained in a gascell. The aim is to produce a photoionised plasma with a simple geometry, 
known ion number density and quantified X-ray heating flux. Absorption spectra from this well characterised plasma will be obtained, using the Z-pinch as a backlighter. This work builds on a previous 'proof of principle' experiment(1). In this way, we hope to obtain a dataset which can be used to test the accuracy of atomic data and simulation codes. The photoionised nature of the plasma makes this data likely to be useful to the astrophysics community.

\section{Simulations}

The model of the gascell behaviour was constructed in an incremental fashion using several simulation codes to analyse the radiation environment at the gascell, simulate the hydrodynamic behaviour of the gascell, and model the atomic kinetic behaviour to produce synthetic emission and absorption spectra. The results of the modelling help us to gain an understanding of the behaviour of the system and allow us to investigate the role of key experimental parameters, helping to assist the experimental design.

\subsection{Radiation environment}

The first part of the model is to characterise the X-ray flux incident on the gascell from the collapsing Z-pinch. The total heating flux has contributions from both the Z-pinch itself and re-radiated emission from parts of the experimental setup such as the return current cannister and anode plate. This part of the model is a key element, as the gascell is heated solely by the radiation field and the hydrodynamic and atomic kinetic behaviour are directly dependent on its characteristics.

The radiation environment is modelled using the Visrad view factor code(4). Based on the specified geometry and material emission properties, the code calculates the radiation re-emitted from each surface of the setup in response to a user specified X-ray radiation source. The geometry used in the model is based on the experimental parameters as far as possible. The Zpinch radiates as a black-body with a Planckian energy distribution, and a radius-power time history inferred from an earlier experiment (shot \#z541). The Z-pinch emission is characterised by a $60 \mathrm{~ns}$ run-in phase, reaching a peak power of $160 \mathrm{TW}$ at $100 \mathrm{~ns}$ with a colour temperature of $200 \mathrm{eV}$ and a pinch radius of $1.1 \mathrm{~mm}$; the majority of the X-ray emission occurs between 95 $105 \mathrm{~ns}$. A detector was positioned at $7 \mathrm{~cm}$ from the pinch axis to represent the front surface of the gascell. A shielding aperture between pinch and gascell was used in one of the model configurations. This allows us to reduce the contribution of re-radiated X-ray components to the total flux arriving at the gascell, making the energy distribution closer to being Planckian. This is desirable since for each Z-pinch shot, the pinch energy spectrum is measured and by allowing the gascell to see only the pinch we can use this information directly to assist analysis of spectra from the gascell.

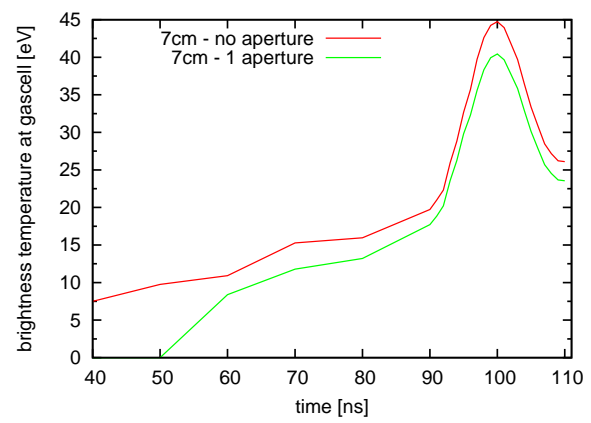

Fig. 1 Brightness temperature at the gascell front surface with and without aperture in place.

The total net flux arriving at the centre of the gascell front surface as a function of time is shown in fig. 1. The flux is expressed in terms of radiation brightness temperature, $\mathrm{T}_{\mathrm{B}}$, using the Stefan-Boltzman law $\mathrm{J}_{\text {tot }}=\sigma \mathrm{T}_{\mathrm{B}}^{4}$ where $\mathrm{J}_{\text {tot }}$ is the total energy-integrated flux arriving at the surface $\left(\mathrm{Wcm}^{-2}\right)$. The model predicts peak values of $\mathrm{T}_{\mathrm{B}}$ of around 40 and $45 \mathrm{eV}$ (corresponding to $2.5 \times 10^{8}$ and $4.5 \times 10^{8} \mathrm{Wcm}^{-2}$ of X-ray power) with and without the aperture respectively.

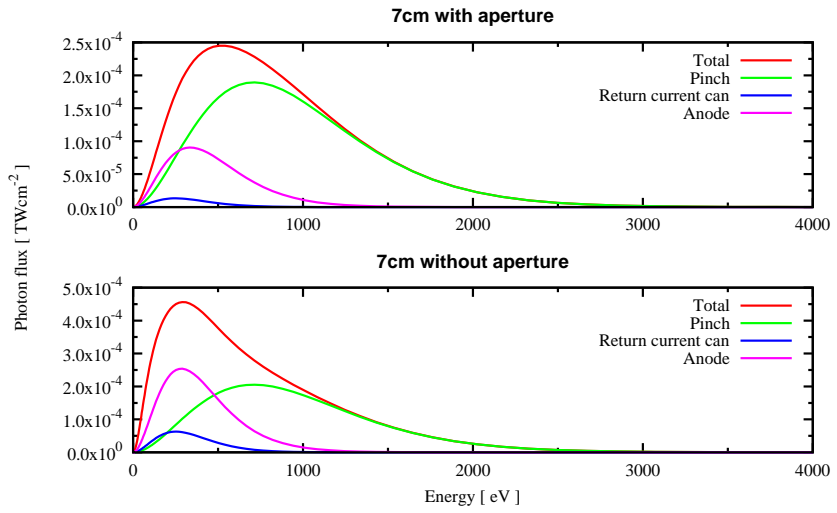

Fig. 2 Composition of the total X-ray flux, showing directly observed pinch radiation and the main re-radiated contributions from return current cannister and anode.

Fig. 2 shows the energy resolved flux components from the pinch, anode and return current cannister with and without aperture at 100ns. With the aperture in place, anode and return current cannister contributions are reduced by a factor of $\sim 3$ with the overall flux reduced by a factor of around 2. In each case, there is almost the same contribution from the pinch. These results show that carefully designed shielding may be 
used to reduce the contribution of re-radiated X-rays at the gascell at the expense of reducing the overall heating flux by a factor of $\sim 2$.

\subsection{Hydrodynamic behaviour}

The time history of the energy resolved heating flux was used to drive a hydrodynamic simulation of the gascell using the Helios-CR code(5). A 1-dimensional model was used, with $1.4 \mu \mathrm{m}$ Mylar walls enclosing a $1 \mathrm{~cm}$ length of neon gas with an ion density of $10^{18} \mathrm{~cm}^{-3}$ (30 Torrs). The heating X-ray flux was applied to one side of the system. LTE opacity lookup tables and Propaceos equation of state data were used for the neon and Mylar regions. A multiangle radiation transport model was used to account for the radiation propagation charateristics. Results presented here correspond to a gascell with a front surface at $7 \mathrm{~cm}$ from the $\mathrm{Z}$-pinch without an aperture in place.

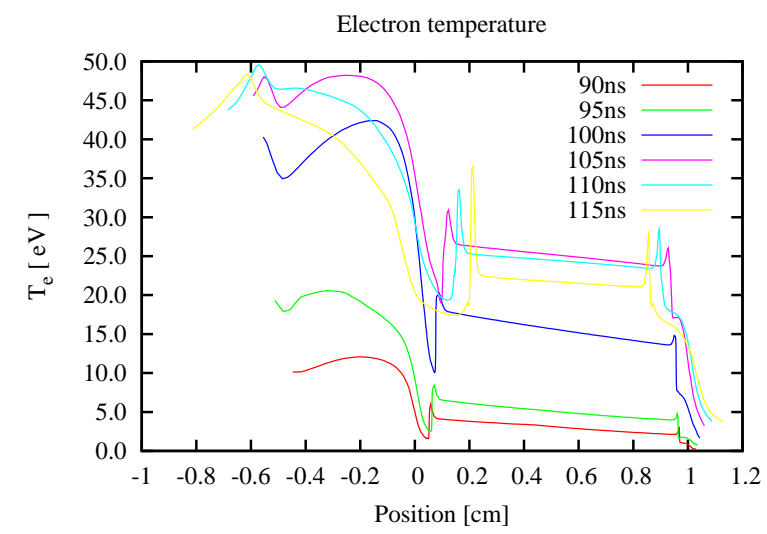

Fig. 3 Space resolved electron temperature in the gascell.

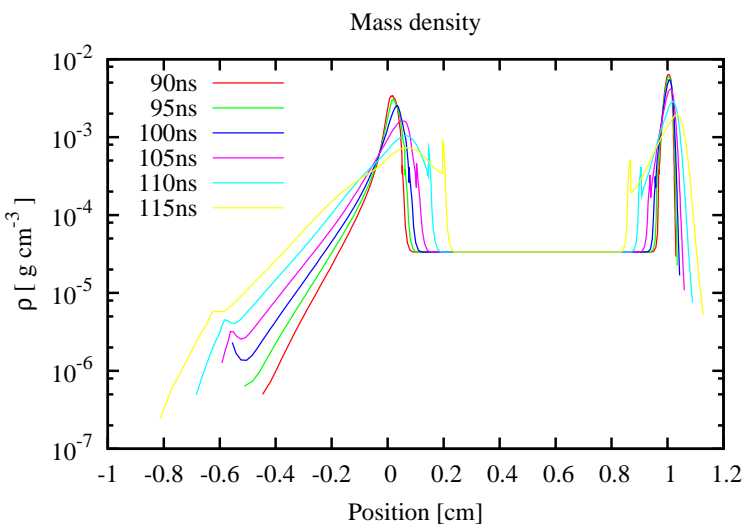

Fig. 4 Space resolved mass density in the gascell.

Figs. 3 and 4 show space resolved temperature and mass density behaviour from the model for times near to the peak of the heating flux (100ns). The Mylar walls are initially at 0 and $1 \mathrm{~cm}$ with the gas in between. Approximately $70 \%$ of the incident radiation field is absorbed by the first Mylar window, leading to heating and ablation expansion of the material into the surrounding vacuum and into the gas region. A smaller proportion of the unattenuated radiation is absorbed by the neon gas and by the rear Mylar window. Shock waves are launched into the neon gas at each Mylarneon interface, leading to a compression of the gas (fig. 4). The central region of the neon is unperturbed by the expanding Mylar near the peak of the radiation drive, with the density remaining largely uniform and the velocities negligible. The temperature profile in the neon is also quite uniform, typically varying less than $2 \mathrm{eV}$ along the full length of the unperturbed region and reaching a maximum of around $25 \mathrm{eV}$ around $5 \mathrm{~ns}$ after the radiation drive peak. At the times of spectroscopic interest, we therefore expect the neon region to be characterised by largely uniform conditions.

The ionisation parameter measures the relative importance of the collisional and photoionisation processes in the plasma. Here we use an ionisation parameter defined by $\xi=4 \pi \mathrm{I} / \mathrm{N}_{\mathrm{e}}\left(\mathrm{erg} \mathrm{cm} \mathrm{s}^{-1}\right)$ where $\mathrm{N}_{\mathrm{e}}$ is the electron number density and I the ionising flux of radiation(1). At the time of peak Z-pinch radiation, in the centre of the neon region we obtain $\xi$ values of $\sim 2.5-3 \mathrm{erg} \mathrm{cm} \mathrm{s}^{-1}$. We also estimate that the hydrogen to helium radiative recombination exceeds the 3 -body recombination rate by a factor of around $10^{4}$, suggesting that the neon plasma is indeed photoionised. Additional simulations found that strategies such as moving the gascell closer to the Z-pinch, reducing the mylar wall thickness and decreasing the gas density, can increase the ionisation parameter by a factor of 3 or more. We note that the degrees of photoionisation found here are small compared with those of active galactic nuclei with similar neon column densities $\left(\sim 10^{18} \mathrm{~cm}^{-2}\right)$, where $\xi$ values of around $2500 \mathrm{erg} \mathrm{cm} \mathrm{s}^{-1}$ have been inferred(6).

The thickness and composition of the front window is a particularly important factor in determining the overall temperature and ionisation parameter characteristic of the neon; a thin, low density window material with high X-ray transmission is desirable to provide the maximum energy for deposition into the gas. On the other hand, the window must be robust enough to withstand the gas pressure without excessive 'ballooning' of the surface and risk of bursting. A series of Helios-CR simulations were carried out, using gascell windows of Be, $\mathrm{C}$ and $\mathrm{CH}$ with thicknesses typically available; bursting pressure calculations for these materials were also performed, using a thin membrane model(7). For the fill pressure of 30 Torrs, $1.4 \mu \mathrm{m}$ of Mylar was found to 
provide a reasonable compromise between X-ray attenuation, material strength and thickness and availability. As a practical note, it is important to quantify the deformation of the gascell windows since this determines the areal density of the gas along the spectrometer line of sight, a quantity required for spectroscopic analysis.

\subsection{Spectral emission}

Hydrodynamic data from the Helios-CR simulation were used as input to the Spect3d code (8) in order to construct synthetic spectra. The code solves the system of atomic rate equations self consistently with the radiative transfer equation for a specified plasma distribution and observer line of sight. Solutions may be obtained using either a time-dependent or steady-state approximation. Here, we present absorption spectra in the neon K-shell region, obtained using a line of sight through the entire length of the gascell with the Z-pinch $\mathrm{X}$-ray flux from the Visrad simulation as the the backlighter. The atomic model used includes fine structure for energy levels in the $\mathrm{H}, \mathrm{He}$ and Li-like states, with configuration averaged states for the Be-like and lower charge states.

Fig. 5 shows the absorption spectra from the gascell in the K-shell region (1000-1250eV). The main absorption features are from the He and Li like ion stages; the He-like series K-shell absorption line series is present between 1070 and $1170 \mathrm{eV}$, with the $\mathrm{He} \beta$ line $(1 \mathrm{~s} 3 \mathrm{p} \rightarrow$ $1 \mathrm{~s}^{2}$ ) at $\sim 1072 \mathrm{eV}$ and the $1 \mathrm{~s} 10 \mathrm{p} \rightarrow 1 \mathrm{~s}^{2}$ line at $\sim 1080 \mathrm{eV}$. Li-like satellites to the $\mathrm{He} \beta$ line are present between 1030 and $1050 \mathrm{eV}$, with the $\mathrm{H} \alpha(2 \mathrm{p} \rightarrow 1 \mathrm{~s})$ and $\mathrm{H} \beta$ absorption lines at 1022 and $1211 \mathrm{eV}$. The overall signal strength follows the strength of the backlighting Z-pinch continuum, peaking at 100ns.

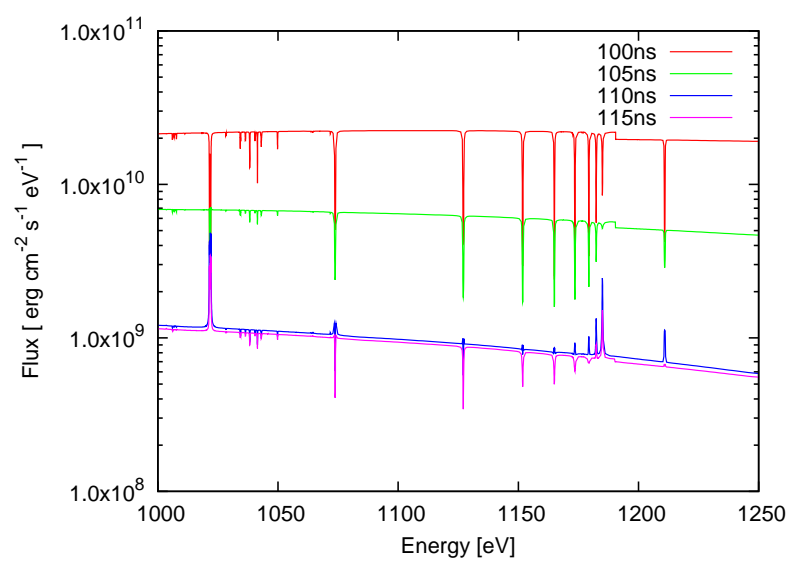

Fig. 5 Absorption spectra in the neon K-shell region from Spect3d

The energy region shown in fig. 5 contains features from three ionisation stages that can be used to assist diagnosis of the plasma from measured spectra. For example, the relative strengths of features from different ion stages are valuable for inferring the charge state distribution whilst comparison of the detailed Li-like satellite structure with computed results provides information the accuracy of the atomic model. We note that these features have been observed experimentally $(1 ; 9)$ and provide the basis for an analysis of the plasma properties currently in progress.

\section{Conclusions}

We have presented results from a modelling study of a photoionised plasma with the aim of aiding the design and understanding of a forthcoming experiment using the Z-pinch facility at Sandia National Laboratories. The aim of the experiment is to produce a well characterised system that can be used to gain insight into atomic-kinetic and radiative properties of photoionised plasmas and provide a test bed for model calculations. The modelling work presented helps us to identify and quantify important experimental parameters such as the radiation environment and the hydrodynamics of the gascell and the interplay between them. Knowlege gained from this study will be valuable for optimising the experimental design and interpreting experimental spectra.

\section{References}

J. E. Bailey et al. Neon photoionization experiments driven by z-pinch radiation. Journal of Quantitative Spectroscopy and Radiative Transfer, 71:157-168, 2001.

M. E. Foord et al. Charge-state distribution and doppler effect in an expanding photoionized plasma. Physical Review Letters, 93:055002, 2004.

P. A. M. Van Hoof et al. Modeling x-ray photoionized plasmas produced at the sandia z-facility. Astrophysics and Space Science, 298:147-153, 2005.

J. J. MacFarlane. Visrada 3-d viewfactor code and design tool for high-energy density physics experiments. Journal of Quantitative Spectroscopy and Radiative Transfer, 81:287-300, 2003.

J. J. MacFarlane and I. E. Golovkin and P. R. Woodruff. Helios-cr a 1-d radiation-magnetohydrodynamics code with inline atomic kinetics modeling. Journal of Quantitative Spectroscopy and Radiative Transfer, 99:381-397, 2006.

A. C. Gonçalves et al. A new model for the warm absorber in ngc 3783: a single medium in total pressure equilibrium. Astronomy and Astrophysics, 451:L23-L26, 2006.

Timoshenko and Woinowsky-Krieger. Theory of Plates and Shells. McGrawHill, 1959. 
J. J. MacFarlane and I. E. Golovkin and P. Wang and P. R. Woodruff and N. A. Pereyra. Spect3d - a multidimensional collisional-radiative code for generating diagnostic signatures based on hydrodynamics and pic simulation output. High Energy Density Physics, 3:181-190, 2007.

J. E. Bailey. Private communication, 2008. 